\title{
Clinical Intervention for Quality Improvement of Gastric-Emptying Studies
}

\author{
Dacian V. Bonta ${ }^{1,2}$, David C. Brandon ${ }^{1,2}$, Jeranfel Hernandez ${ }^{1,2}$, Minesh Patel $^{1}$, Sandra Grant ${ }^{1}$, and Naomi Alazraki ${ }^{1,2}$ \\ ${ }^{1}$ Department of Nuclear Medicine, VAMC Atlanta, 1670 Clairmont Rd., Decatur, Georgia; and ${ }^{2}$ Department of Radiology and \\ Imaging Sciences, Emory University, 1364 Clifton Rd., Atlanta, Georgia
}

Prompted by clinical concerns for false-negative tests, we implemented a clinical intervention consisting of a training session and an image-based verification procedure to document homogeneous radioactivity distribution in the radiolabeled meal (egg substitute per the guideline). Methods: A technologist training session emphasized the importance of thorough mixing of $99 \mathrm{~m}$ Tc-sulfur colloid in the egg meal. For 6 mo after training, an image of the prepared mixed egg was acquired before patient ingestion. Consecutive gastric-emptying studies performed 6 mo before and after training were reviewed by 2 experienced physicians. Results: There were 7 abnormal and 44 normal studies before and 15 abnormal and 29 normal studies after training $(P<0.05)$. Subjective evaluations of images for meal-mixing quality by 2 readers correlated with each other and with an objective measure of expected gastric-emptying physiology (correlation coefficients, 0.54 and 0.38 , respectively). Conclusion: The described clinical intervention improved the accuracy of our gastric-emptying studies by decreasing falsenegative studies.

Key Words: quality improvement; quality assurance; gastricemptying scintigraphy; Tougas protocol

J Nucl Med Technol 2014; 42:274-277

DOI: 10.2967/jnmt.114.146167

$\mathbf{T}$ he solid and liquid components of a meal are handled differently by the stomach $(1,2)$. Solid emptying requires the fragmentation of the food bolus into small particles, resulting in a lag phase, followed by emptying at a fixed rate. On the other hand, liquid emptying starts immediately, resulting in an exponential emptying curve. The procedure described in the joint Society of Nuclear Medicine and Molecular Imaging/American Neurogastroenterology and Motility Society guideline (3) evaluates the emptying of the solid component of a complex meal containing both solid and liquid components.

\footnotetext{
Received Oct. 22, 2014; revision accepted Oct. 23, 2014.

For correspondence or reprints contact: Dacian V. Bonta, VAMC Atlanta, Nuclear Medicine Department (115), 1670 Clairmont Rd., Decatur, GA 30033. E-mail: dvbonta@yahoo.com

Published online Nov. 11, 2014.

COPYRIGHT (c) 2014 by the Society of Nuclear Medicine and Molecular Imaging, Inc.
}

Careful meal preparation results in excellent binding to the solid component of the egg meal (4). However, some authors have observed lower labeling efficiency of the solid egg meal component (5).

In our practice, we primarily use the standardized gastric meal developed by Tougas (6), as recommended by the Society of Nuclear Medicine and Molecular Imaging guideline. Oatmeal and liquid emptying protocols are used only when the Tougas protocol cannot be performed because of patient-related factors. ${ }^{99 \mathrm{~m}} \mathrm{Tc}$-sulfur colloid (18.5-37 MBq [0.5-1 mCi]) and egg substitute (118 mL [4 oz]; Eggbeaters [ConAgra Foods, Inc.]) are mixed in a casserole (Fig. 1). The mixture is cooked in a microwave for $2 \mathrm{~min}$ to firm consistency, with a break at $1 \mathrm{~min}$ for additional mixing, and then served to the patient in the same casserole. We acquire 1-min anterior and posterior images with a dualhead camera at $0 \mathrm{~min}, 30 \mathrm{~min}, 1 \mathrm{~h}, 2 \mathrm{~h}, 3 \mathrm{~h}$, and $4 \mathrm{~h}$. Gastric-retention percentages are computed from the geometric mean of anterior and posterior counts in the region of interest drawn around the stomach. The initial image includes all the abdominal activity, per the guideline. No continuous imaging is acquired. Technologists document whether the patient ingested the meal in the required time (10 min). All patients included in this study consumed the meal in the allotted time (10 $\mathrm{min})$.

During our regular practice using the Tougas protocol, we have noticed gastric-emptying studies with activity present in the gut in the first patient image $(0-1 \mathrm{~min})$. We have also noted studies with a higher emptying percentage from 0 to $30 \mathrm{~min}$ than from 30 to $60 \mathrm{~min}$. These observations are not consistent with expected gastric physiology because the solid component needs to be broken down to sufficiently small size to pass through the pylorus first (lag phase). Thus, more emptying should occur from 30 to $60 \mathrm{~min}$ than from 0 to $30 \mathrm{~min}$. However, these anomalies are easily explained if a significant fraction of the radioactive label was either unbound or weakly bound to the solid component. As a result of improper labeling, part of the ingested activity will exit the stomach according to a liquid emptying pattern (initially faster, then slower). If the radioactivity is not evenly mixed throughout the egg substitute, this would contribute to another cause for erratic and unreliable gastric-emptying measurements, which is the focus of this report. 


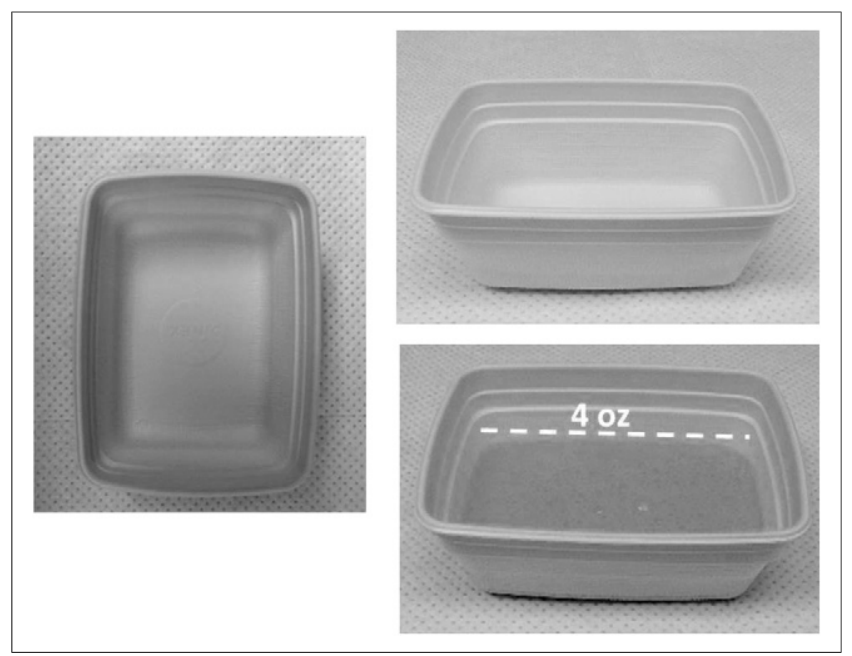

FIGURE 1. Casserole in which egg beater and radioactive label are mixed, cooked/heated in microwave oven, and then served to patient. Shown, casserole with prescribed amount of egg beater.

The egg labeling is a physical-chemical process in which the sulfur colloid is adsorbed onto the egg albumen at increased temperature. As demonstrated in the literature (4), cooking the egg substitute and sulfur colloid mixture to firm consistency will produce a reliable label. Good mixing ensures that camera measurements, which reflect the percentage of radioactivity retained in the stomach, accurately reflect the percentage of the meal retained in the stomach. However, although proper cooking to firm consistency can be assessed easily by visual inspection, the uniform distribution of radioactivity in the prepared meal is more difficult to evaluate. We sought to address suboptimal mixing, a potential weak link in the gastric-imaging chain, through training and supervision. Subsequently, we developed and validated a verification procedure based on the fact that a uniform distribution of radiotracer within the meal will produce a uniform image when the casserole containing the labeled egg substitute is imaged with a nuclear medicine camera.

\section{MATERIALS AND METHODS}

A continuing education session was organized and delivered by our chief technologist to our staff of 10 CNMT specialists of the Nuclear Medicine Department at the Atlanta Veteran Affairs' Medical Center. During the training session, the importance of achieving thorough mixing of the egg substitute with the radioactive label was reinforced. The duration of the mixing and the need for the label to be distributed evenly through the egg substitute were reviewed. Technologists were instructed to place the $118 \mathrm{~mL}$ of egg substitute in the casserole first, followed by injection of the radiopharmaceutical throughout the volume of egg substitute, not just in 1 superficial spot. The mixing was done with a fork, with mixing strokes directed from one edge of the casserole to the other, in a crisscrossing pattern, for at least $1 \mathrm{~min}$. A practical hands-on demonstration was also performed during the session. Before the training session, technologists had only been given an instruction to mix the egg and labeled sulfur colloid thoroughly. There were no changes of personnel during the duration of the study.

After the training session, we also introduced a simple imagingbased verification procedure to evaluate the quality of mixing. The egg component of the meal was imaged after preparation but before presentation to the patient for ingestion. The cooked meal was imaged for $30 \mathrm{~s}$, using the same camera as used for the patient study. This image, together with an image of the container after the patient had eaten the egg component, was saved as a raw image and transferred to the physician's image review station as part of the study. Beyond the addition of the meal-imaging step, the mealpreparation protocol and the patient-imaging protocol remained unchanged from the preintervention procedures.

During the 6 mo after the start of our clinical intervention, we recorded the gastric-emptying percentages for all consecutive patients who consumed the entire meal, for a total of 44 consecutive patient studies (postintervention population). We compared the postintervention results with the 51 consecutive gastric-emptying studies using the Tougas meal acquired in the 6 mo before the training session (preintervention population). There were no significant differences between the pre- and postintervention population with respect to age, sex, or diabetes mellitus diagnosis (Table 1). The patient population for both before and after intervention comprised veterans of the greater Atlanta area. The institutional review board approved this retrospective study, and the requirement to obtain informed consent was waived.

To assess whether changes in mixing thoroughness evaluated by imaging can be related to the observed gastric-emptying patterns, we performed an image interpretation experiment on the dataset of 44 meal images and their associated gastricemptying curves. We used the difference between the gastric emptying from 0 to $30 \mathrm{~min}$ and the emptying from 30 to $60 \mathrm{~min}$ as an objective measure of whether gastric emptying was physiologic.

Two experienced nuclear medicine physicians independently evaluated the quality of the meals' mixing by visual inspection of

TABLE 1

Patient Characteristics for Pre- and Postintervention Groups

\begin{tabular}{lccc}
\hline \multicolumn{1}{c}{ Patient characteristic } & Before intervention & After intervention & Statistical significance \\
\hline Mean age \pm variance $(\mathrm{y})$ & $54 \pm 9$ & $56 \pm 10$ & $P>0.05$ \\
Sex & 32 & 26 & $P>0.05$ \\
$\quad$ Male & 19 & 18 & \\
Female & 26 patients & 23 patients & $P>0.05$ \\
Diabetes mellitus diagnosis & & \\
\hline
\end{tabular}


the meal images and ranked the images from the best mixed to the worst mixed. The readers were masked to the observed gastricemptying percentages corresponding to each meal.

The studies were also ranked by the difference in measured gastric emptying between 30 and $60 \mathrm{~min}$ and between 0 and $30 \mathrm{~min}$, considered an objective evaluation of gastric-emptying physiology (objective ranking). The pairwise Pearson rank correlation among the 3 rankings ( 2 readers and the gastricemptying result) was computed.

\section{RESULTS}

In the preintervention patient population, there were 7 (14\%) studies with abnormal (10\% or more) gastric retention at $4 \mathrm{~h}$ and 44 studies with normal (less than $10 \%$ ) gastric retention at $4 \mathrm{~h}$. In the postintervention population, there were 15 (34\%) studies with abnormal gastric retention at $4 \mathrm{~h}$ and 29 studies with normal gastric retention at $4 \mathrm{~h}$. The difference between these proportions corresponds to a $P$ value of less than 0.05 , by both Pearson $\chi^{2}$ with Yates correction for continuity and by 2-tailed Fischer exact probability test, computed using an online calculator (7).

The Pearson rank correlation coefficient between each of the 2 readers ranking the studies by perceived quality of mixing and ranking the studies by the difference between 0 - to 30 -min and 30- to 60-min emptying were 0.36 and 0.40 (perfect correlation, 1; no correlation, $0 ; P<0.05$ for difference from 0$)$. The Pearson rank correlation coefficient between the 2 physicians' rankings was $0.54(P<0.05$ for difference from 0 ). Thus, studies perceived as better mixing were also more likely to display the expected pattern of solid emptying (less emptying from 0 to $30 \mathrm{~min}$ than from 30 to $60 \mathrm{~min}$ ).

After completing the ranking process, the 2 physicians reviewed the rankings in light of the objective measure findings. The indicator of good mixing was the presence of more uniform (even if lumpy bumpy) distribution of activity throughout the entirety of the casserole, including close to the edge of the casserole. Indicators of poor mixing were the presence of large regions of different radiotracer activity, in particular, the presence of significant areas of decreased radiotracer activity toward the edges. Figure 2 illustrates these findings.

\section{DISCUSSION}

The diagnostic accuracy of scintigraphic evaluation for delayed gastric emptying is dependent on good meal quality. The radioactive tagged sulfur colloid will be confined to the digestive tract, whether bound to the solid component or not. Thus, we cannot use altered biodistribution as a quality control as for other nuclear medicine studies (e.g., visualization of thyroid in gastrointestinal bleeding studies). Altered biokinetics (in our case, early visualization of small bowel, more gastric emptying from 0 to $30 \mathrm{~min}$ than from 30 to $60 \mathrm{~min}$ ) may suggest technical artifact, but the interpretation of altered biokinetics is made difficult by

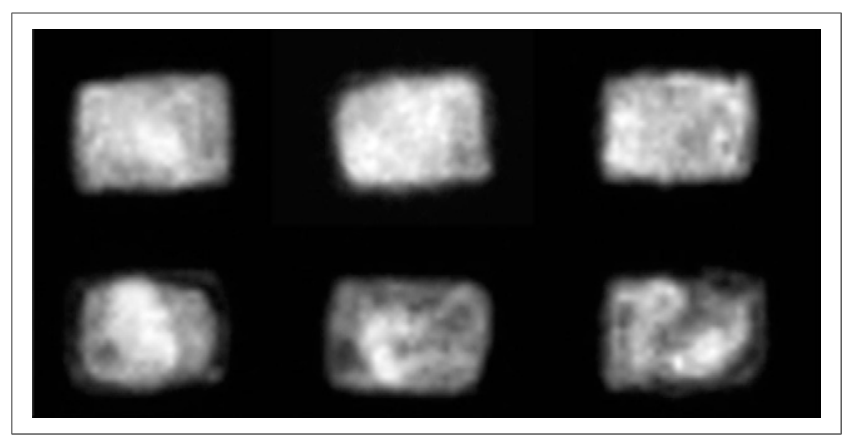

FIGURE 2. (Top) Well-mixed meals, which correlated to expected solid-meal emptying pattern (less emptying from 0 to $30 \mathrm{~min}$ than from 30 to $60 \mathrm{~min}$ ). (Bottom) Poorly mixed meals, which correlated to unexpected solid-meal emptying pattern (more emptying from 0 to $30 \mathrm{~min}$ than from 30 to $60 \mathrm{~min}$ for liquid emptying pattern).

interpatient variability in anatomy, physiology, and disease status.

The egg meal is a semi-solid preparation. As a result, its properties (both degree of label binding to the solid component and the uniformity of radioactivity density in the solid component) may vary from point to point. Existing literature (4) demonstrates that the binding is appropriate when radioactivity and eggs are well mixed and the meal is cooked properly. On the other hand, if the prepared gastric meal is inhomogeneous, the properties of the sample tested may differ significantly from the properties of the part of the meal ingested by patients. Thus, testing a sample would not be informative about the properties of the remainder of the meal. Conversely, imaging the meal before administration to a patient could help identify this pitfall, as we described in the second half of the paper. Patient-related factors (e.g., stomach position, abdominal geometry, the movement of the food bolus from the fundus to the body and antrum, and the presence and severity of gastroparesis) may act as confounders. However, these confounders are independent of the patient receiving a well- or poorly mixed test meal.

The Tougas protocol is relatively resilient against labeling variations. For example, assume that only $50 \%$ of the radioactivity is tagged to the solid component of the meal. Even then, only patients with actual 4-h retention between $10 \%$ and $20 \%$ would be incorrectly labeled as normal (false-negative). Patients with more than $20 \%$ retention of the solid component will still be correctly classified as abnormal (true-positive). On the other hand, a 2-h protocol that assumes retention of less than 50\% of the meal as normal would classify almost all subjects as normal, except perhaps those patients with severe gastroparesis who also have markedly delayed liquid-component emptying.

Additional criteria proposed for normal or abnormal gastric retention would allow the test to be stopped at $2 \mathrm{~h}$, except for a few patients who have borderline 2-h gastricretention values, between the normal and the abnormal 
thresholds (8). Reliable, good-quality labeling is paramount to the success of any such attempt to use the 2-h time point to diagnose delayed gastric emptying.

\section{CONCLUSION}

To address a suspected pitfall in our clinical practice, we have implemented an intervention to improve the preparation (mixing) of the gastric-emptying meal. The intervention consisted of a continuing education session, followed by the introduction of an image-based verification procedure. After intervention, we observed a significant increase in the percentage of abnormal studies, compared with the pretest percentages, suggesting that improper meal-mixing was a problem in our practice. Better mixing, as assessed by visual inspection, was correlated with improved quality of gastric-emptying studies as evaluated by an objective measure of gastricemptying physiology. Therefore, visual assessment of meal for thoroughness of mixing provides an objective basis for evaluating the quality of gastric-emptying meal preparation.

\section{DISCLOSURE}

No potential conflict of interest relevant to this article was reported.

\section{REFERENCES}

1. Siegel JA, Urbain JL, Adler LP, et al. Biphasic nature of gastric emptying. Gut. 1988;29:85-89.

2. Stacher G, Bergmann H. Scintigraphic quantitation of gastrointestinal motor activity and transport: oesophagus and stomach. Eur J Nucl Med. 1992;19: 815-823.

3. Donohoe KJ, Maurer AH, Ziessman HA, et al. Society for Nuclear Medicine; American Neurogastroenterology and Motility Society. Procedure guideline for adult solid-meal gastric-emptying study 3.0. J Nucl Med Technol. 2009;37:196-200.

4. Knight LC, Kantor S, Doma S, et al. Egg labeling methods for gastric emptying scintigraphy are not equivalent in producing a stable solid meal. J Nucl Med. 2007;48:1897-1900.

5. Shuter B, Ng SM. Radiolabelling of egg-meals for gastric emptying studies: a comparison of ${ }^{99 \mathrm{~m}} \mathrm{Tc}$ sulfur and ${ }^{99 \mathrm{~m}} \mathrm{Tc}$ stannous colloids. Nucl Med Commun. 2005;26:369-374

6. Tougas G, Chen Y, Coates G, et al. Standardization of a simplified scintigraphic methodology for the assessment of gastric emptying in a multicenter setting. Am J Gastroenterol. 2000;95:78-86.

7. For a $2 \times 2$ Contingency Table. http://faculty.vassar.edu/lowry/tab2x2.html. Accessed on November 7, 2014.

8. Bonta DV, Lee HY, Ziessman HA. Shortening the 4-hour gastric-emptying protocol. Clin Nucl Med. 2011;36:283-285. 